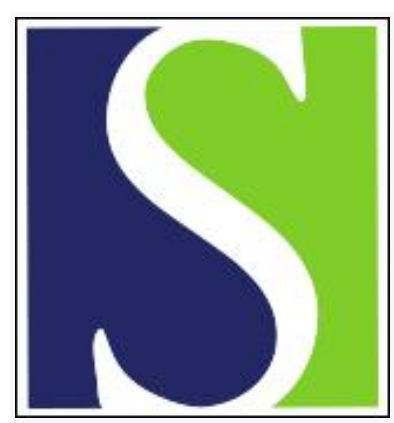

Scand J Work Environ Health 1986;12(4):280-283

https://doi.org/10.5271/sjweh.2140

Issue date: Aug 1986

Pathological changes observed in the finger biopsy of patients with vibration-induced white finger.

by Takeuchi T, Futatsuka M, Imanishi H, Yamada S

This article in PubMed: www.ncbi.nlm.nih.gov/pubmed/3775312

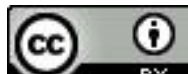




\title{
Pathological changes observed in the finger biopsy of patients with vibration-induced white finger
}

\author{
by Tadao Takeuchi, MD, Makoto Futatsuka, MD, Hayao Imanishi, MD, Shinya Yamada, MD
}

\begin{abstract}
TAKEUCHI T, FUTATSUKA M, IMANISHI H, YAMADA S. Pathological changes observed in the finger biopsy of patients with vibration-induced white finger. Scand $J$ Work Environ Health 12 (1986) 280-283. Lesion biopsies of 60 fingers from 30 patients with vibration-induced white finger (VWF) and control observations from seven fingers of five referents demonstrated three main characteristic pathological changes. First, in each case, the muscular layers of the arteries revealed intense thickening with strong hypertrophy of individual muscle cells without intimal fibrosis. Periarterial fibrosis was also noted. Arteriosclerosis with foamy cells, lipid deposition, and fibrous sclerosis was occasionally observed. The second main change was demyelinating neuropathy in the peripheral nerves in which a marked loss of nerve fibers had occurred. There was also an increase in the number of Schwann cells and fibroblasts with strong collagen formation. Severe loss of myelin sheath frequently occurred, and relatively smaller axons without myelin which appeared to have regenerated were observed. Perineural fibrosis was also noted. In the third main pathological change connective tissues with collagen were increased not only in the perivascular and perineural lesions, but also abundantly in the corium of the skin. The elastic fibers there had been destroyed. These three principal pathological changes are very useful for the histopathological diagnosis of VWF.
\end{abstract}

Key terms: muscular hypertrophy, vibration neuropathy, VWF.

Our knowledge of the pathology of vibration-induced white finger (VWF) is very poor, in spite of many studies on its clinical and pathophysiological aspects. Ashe et al (1) pointed out medial muscular hypertrophy of small arteries in their pathological descriptions of arterial biopsies from two persons with VWF, of which one, 42 years of age, revealed medial muscular hypertrophy with no particular tendency for hyalination and the other, 40 years of age, had advanced muscular hypertrophy together with moderate to marked subintimal fibrosis. There was, however, no description of the nerve and other tissues. Walton (7) reported vascular changes in the arteries resembling those of the second case of Ashe et al (1) in his one autopsy case, and he described no special findings in the peripheral nerves except for a small infiltration of round cells in the end organs. On the other hand Karpova (2) has experimentally demonstrated demyelinated and Wallerian degenerations in the peripheral nerves of rabbits.

Over the past few years, we have investigated pathological correlates of behavioral syndromes which were apparently caused by the use of vibrating tools (4). In the present report, we describe the pathological findings in focal lesions of 60 finger biopsies obtained from 30 patients in comparison with those of seven fingers of five persons without experience with such tools.

1 Kumamoto University Medical School, Imanishi Hospital, and the Nagoya University School of Medicine, Japan.

Reprint requests to: Dr T Takeuchi, Kumamoto University Medical School, 2-2-1 Hongo, Kumamoto 860, Japan.

\section{Subjects and methods}

The lesion biopsies of 60 fingers from 30 patients with VWF and of seven fingers from five persons as a reference were performed with the approval of the individuals concerned. The patients had had experience with chain saws (12 cases), pneumatic hammers (10 cases), or both tools ( 8 cases). All the patients were males aged 30 to 70 years, mean 53 (SD 9) years. The exposure to vibrating tools had lasted 5 to 23 years, mean 17 (SD 6) years. At the time of the biopsy, the individual patients suffered from vibration syndrome with Raynaud's phenomenon. The grade of VWF was severe in 26 cases and mild in four cases.

The tissue samples obtained by punch biopsy were fixed in Bouin's mixture and $10 \%$ neutral formalin at $4^{\circ} \mathrm{C}$. Usual cut sections from paraffin-embedded blocks were subjected to staining with hematoxylineosin stain, elastica-v Gieson stain, Azan trichrome stain, silver impregnation, Kluver-Barrera stain, and Bodian stain.

\section{Results}

It was found from the results of the double finger biopsy of these patients that there were three main types of pathological changes in the fingers and that these findings could be confirmed in every case.

The first type of changes was vascular, particularly lesions of the artery walls in the small arteries and of the arterioles in the fingers. An increase in the thickness of the whole arterial wall was noted (figures $1-3$ ). In particular, the thickness of the walls resulted from muscular hypertrophy, which was morphologically identified by the presence of hypertrophied nuclei and 
Table 1. Pathohistological changes in small arteries of the subjects. (VWF $=$ vibration-induced white finger)

\begin{tabular}{|c|c|c|c|c|c|c|}
\hline \multirow{2}{*}{ Pathological change } & \multicolumn{4}{|c|}{ Biopsies from VWF patients ${ }^{a}(N=60)$} & \multicolumn{2}{|c|}{ Biopsies from referents ${ }^{a}(N=7)$} \\
\hline & Grade 0 & Grade 1 & Grade 2 & Grade 3 & Grade 0 & Grade 1 \\
\hline Increase of adventitia & - & 5 & 22 & 33 & 7 & - \\
\hline $\begin{array}{l}\text { Thickening of media } \\
\text { Hypertrophy of muscle } \\
\text { Increase of collagen }\end{array}$ & $\overline{36}$ & $\begin{array}{r}2 \\
24\end{array}$ & 9 & $\frac{49}{-}$ & $\begin{array}{l}7 \\
7\end{array}$ & $\overline{-}$ \\
\hline $\begin{array}{l}\text { Destruction of internal elastic } \\
\text { layer ( } T \text { elastica interna) }\end{array}$ & 54 & $1^{\mathrm{b}}$ & $2^{b}$ & $3^{b}$ & 7 & - \\
\hline Intimal sclerosis & 39 & $17^{\mathrm{b}}$ & $4^{b}$ & & 4 & $3^{\mathbf{b}}$ \\
\hline
\end{tabular}

a Grade $0=$ no change, grade $1=$ minor change, grade $2=$ moderate change, grade $3=$ severe change. NOTE: $3=$ the most intensely increased thickness of the musle layer of about threefold or more, $2=$ a moderately increased thickness of about twice or more, $1=$ increased thickness of less than twice.

b With arteriosclerosis.

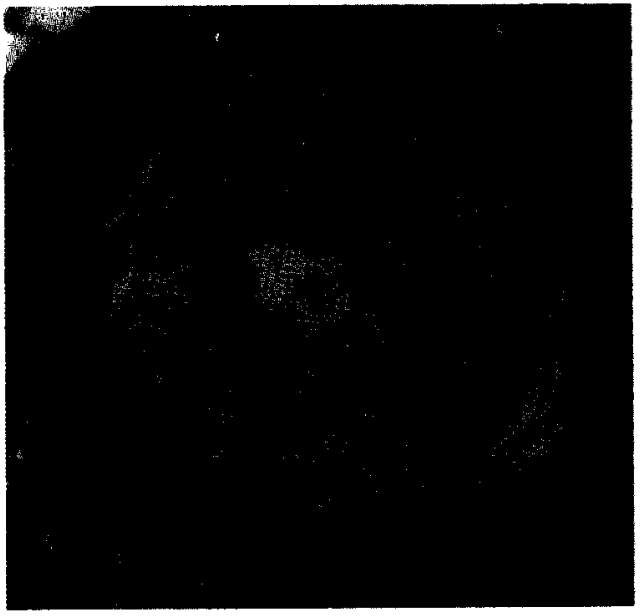

Figure 1. Thickening of a small artery with medial muscular hypertrophy and perivascular fibrosis. (56-year-old male, Elastica-v Gieson stain, $200 \times$ )

an increased cytoplasmic bulk. There was no significant fibrosis in the muscular layer or in the intimal layer, but adventitial and perivascular fibrosis was usually present. Such vascular changes occurred frequently and strongly in the larger parts of small arteries and became weaker in the smaller parts of small arteries, as well as in the arterioles.

We classified the increased thickness of the muscle layer into three degrees, and the results obtained from the 60 biopsied fingers are summarized in table 1. All cases revealed an increased thickness of the muscular layer, and the smaller arteries were severely $(82 \%)$ to moderately $(15 \%)$ thickened. Adventitial or perivascular fibrosis also occurred frequently and severely $(55 \%)$ or moderately $(37 \%)$. There were no cases without perivascular fibrosis. Intimal fibrosis was observed in 21 cases ( $35 \%$ ), and most of these cases were minor. There were only four cases with moderate changes of intimal fibrosis. Such intimal fibrosis was rather a complication of arteriosclerosis with foamy

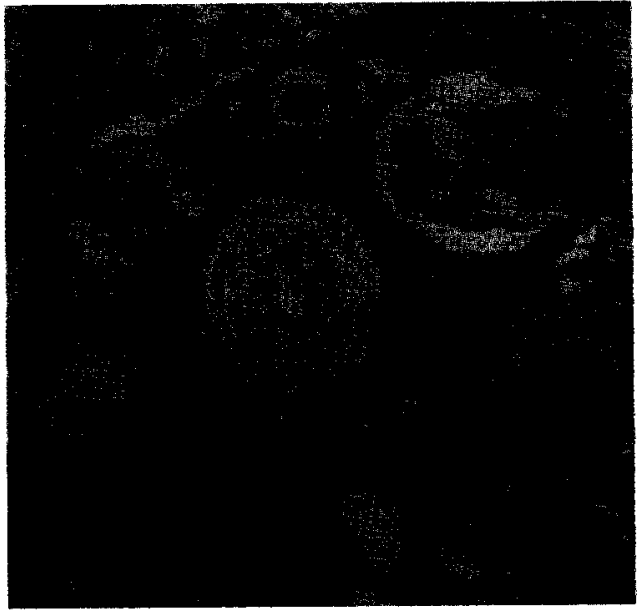

Figure 2. Thickening of smaller arteries with medial muscular hypertrophy and perivascular fibrosis. (30-year-old male, Elastica-v Gieson stain, $200 \times$ )

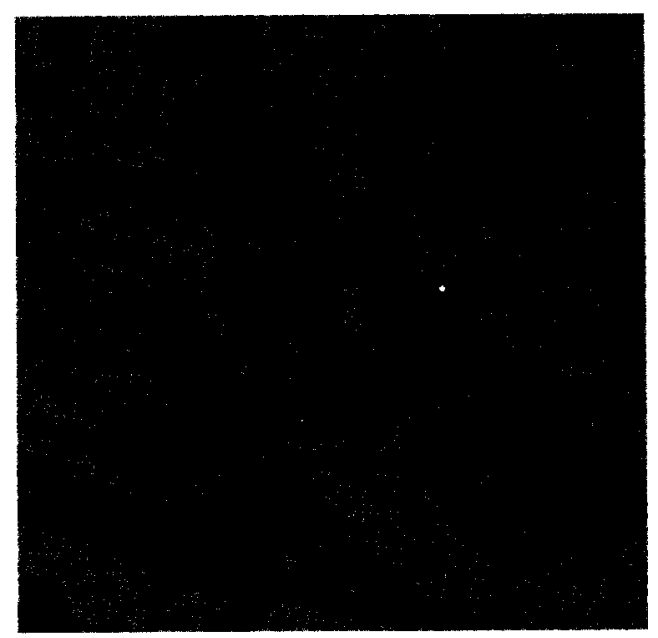

Figure 3. Thickening of arterioles with muscle hypertrophy and perivascular fibrosis. (55-year-old male, Elastica-v Gieson stain, $200 x$ ) 
Table 2. Pathohistological changes in peripheral nerves of the subjects. (VWF = vibration-induced white finger)

\begin{tabular}{|c|c|c|c|c|c|c|c|}
\hline \multirow{2}{*}{ Pathological change } & \multicolumn{4}{|c|}{ Biopsies from VWF patients ${ }^{a}(N=60)$} & \multicolumn{3}{|c|}{ Biopsies from referents ${ }^{a}(N=7)$} \\
\hline & Grade 0 & Grade 1 & Grade 2 & Grade 3 & Grade 0 & Grade 1 & Grade 2 \\
\hline Loss of myelin & - & - & 16 & 44 & 1 & 6 & - \\
\hline Disappearance of axon & - & 12 & 34 & 14 & 7 & - & - \\
\hline Increase of Schwann cells & - & - & 14 & 46 & 1 & 6 & - \\
\hline $\begin{array}{l}\text { Increase of collagen } \\
\text { Increase of perineural }\end{array}$ & - & 2 & 15 & 43 & - & 7 & - \\
\hline connective tissue & - & 2 & 22 & 36 & 2 & 4 & 1 \\
\hline
\end{tabular}

a Grade $0=$ no change, grade $1=$ minor change, grade $2=$ moderate change, grade $3=$ severe change. NOTE: $3=$ the most severe destruction of the myelin sheath with disappearance of two-thirds or more in number of the nerve fibers, $2=$ moderate disappearance of about half of the neve fibers, $1=$ disappearance of about one-third of the nerve fibers.

Table 3. Pathohistological changes in connective tissues of the subjects. (VWF = vibration-induced white finger)

\begin{tabular}{|c|c|c|c|c|c|c|}
\hline \multirow{2}{*}{ Pathological change } & \multicolumn{4}{|c|}{ Biopsies from VWF patients ${ }^{a}(N=60)$} & \multicolumn{2}{|c|}{ Biopsies from referents ${ }^{a}(N=7)$} \\
\hline & Grade 0 & Grade 1 & Grade 2 & Grade 3 & Grade 0 & Grade 1 \\
\hline Increase of collagen & - & 1 & 16 & 43 & 1 & 6 \\
\hline $\begin{array}{l}\text { Destruction and disappearance } \\
\text { of elastic fibers }\end{array}$ & - & 3 & 23 & 34 & 7 & - \\
\hline Change of reticulin fibers & - & 5 & 40 & 15 & 7 & - \\
\hline
\end{tabular}

a Grade $0=$ no change, grade $1=$ minor change, grade $2=$ moderate change, grade $3=$ severe change.

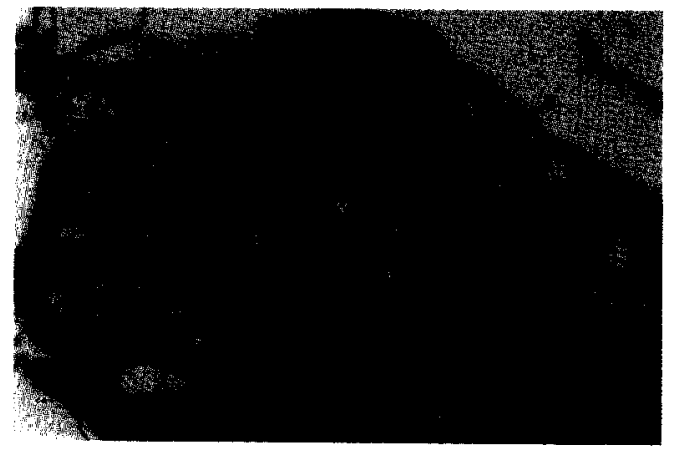

Figure 4. Severe neuropathy with loss of nerve fibers and increased collagen in the endoneurium and perineurium. Perineural fibrosis is also visible in this cross-cut section. (53-yearold male, Azan stain, $200 \times$ )

cells or lipid deposition in the focal lesions. The thickness of the arteriolar walls was increased, mainly as a result of hypertrophy of the muscular layer with slight perivascular fibrosis. The capillary network in the corium appeared to be sparsely scattered, and the capillaries were decreased in number.

The second type of changes involved the peripheral nerves. Axons were decreased in number and sometimes disappeared. Smaller axons not usually seen under normal conditions were relatively increased, whereas the myelin sheath disappeared. The peripheral nerve fibers were thereby decreased in number and were replaced by increased collagen (figures 4 and 5). Destruction of the myelin sheath in two-thirds or more of the nerve fibers occurred in $73 \%$ of the cases, and in the remaining cases $(27 \%)$ the destruction was

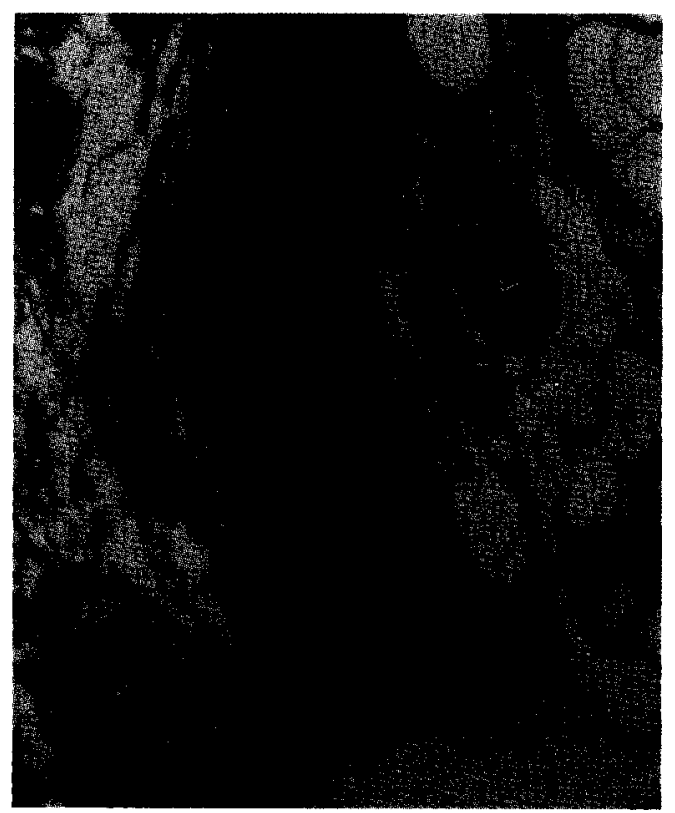

Figure 5. Neuropathy with severe loss of nerve fibers and increased Schwann cells and collagen in a longitudially cut section. (46-year-old male, Azan stain, $200 \times$ )

moderate. On the other hand, the destruction and disappearance of axons occurred less severely and less frequently than the loss of myelin. The loss of twothirds of the axons was observed in only $23 \%$ of the cases (table 2). Schwann cells were always increased in number, and fibroblasts with collagen were increased not only in the endoneurium but also in the perineurium. In particular, a circular increase was 
noted in the connective tissue in the perineural area. In the pacinian corpuscles, the axons sometimes disappeared, and periaxonal nuclei were frequently increased.

The third main type of change was noted in the connective tissues of the skin, especially in the corium, as well as in the perivascular and perineural regions (table 3). Collagen was always greatly increased (figure 6). Elastic fibers were frequently destroyed and disappeared in the corium. The perivascular or pericapillary reticulum fibers were usually decreased.

\section{Discussion}

It is well known that Raynaud's phenomenon occurring in association with the occupational use of vibrating tools is intimately related to the intense contraction of local arteries. Medial muscular hypertrophy with an increase in the thickness of the arterial walls presumably plays an important role in constricting the small arteries more intensely than normally. Strong muscular hypertrophy as described first by Ashe et al (1) was observed in all the fingers of our patients, and such intense thickness of the muscular layers was not identified in the referents. Intimal fibrosis and hyalinosis did not appear to be related to vibration exposure, since the muscular hypertrophy was not always accompanied by such fibrosis. It was considered on the basis of the pathological findings that the intimal fibrosis and hyalinosis represented a complication of occasional arteriosclerosis with foamy cells, lipid deposition, or fibrous sclerosis. Perivascular fibrosis with abundant collagen was found for the first time in the present study. Such collagen formation presumably resulted from perivascular edema which could increase as a result of repeated vibration exposure.

The clinical signs and symptoms of VWF suggest the presence of polyneuropathy $(3,6)$ although Walton (7) was unable to identify any changes of neuropathy. Karpova (2) suggested its occurrence from experimental data on rabbit neuropathy. The material from human subjects obtained from a large number of finger biopsies in the present study revealed that peripheral nerves in the fingers of patients with VWF were always disturbed. Loss of myelin sheath following demyelination was the main observation. A loss of axon cylinders was also noted, but less frequently than the loss of myelin. There were extremely fine axons which possibly had resulted from the regeneration of previously disturbed axons. Such fine axons were difficult to stain by silver impregnation, but they could be observed under the electron microscope (5). We were particularly interested in the peculiar finding of marked perineural fibrosis. Thick circular fibrosis increasing the thickness of the perineurium or epineurium was noted in cross-cut sections. Such perineural fibrosis, as well as perivascular fibrosis, appeared to be characteristic of

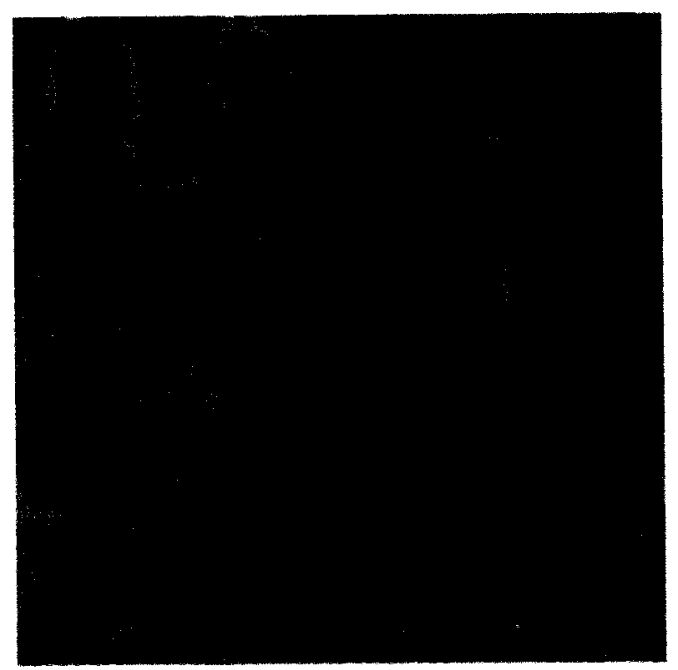

Figure 6. Abundant formation of collagen bundles including an increase in collagen fibers and the destruction of elastic fibers. (48-year-old male, Elastica-v Gieson stain, $200 \times$ )

VWF, and these forms of fibrosis were presumably due to previously formed edema that possibly resulted from the strong physical power of the vibration. A similar mechanism could also have caused the increase of collagen in the corium of the skin where the elastic fibers were gradually destroyed.

Statistically, a positive correlation (r) was noted between the vibration exposure period and the three main pathological changes $(r=0.292)$, in particular among chain-saw operators. In addition clinically severe cases revealed significantly $(p<0.01)$ intense pathological changes in the peripheral arteries, nerves, and corium of the skin.

\section{References}

1. Ashe WF, Cook WT, Old JW. Raynaud's phenomenon of occupational origin. Arch Environ Health 5 (1962) $333-343$.

2. Karpova NI. Vibration and nervous system. Leningrad Medicine, Leningrad 1976, pp 66-147.

3. Lukas E. Peripheral nervous system and hand-arm vibration exposure. In: Brammer AJ, Taylor W, ed. The vibration effects on the hand and arm in industry. John Wiley \& Sons, New York, NY 1982, pp 39-44.

4. Takeuchi T, Imanishi H. Histopathologic observations in finger biopsy from thirty patients with Raynaud's phenomenon of occupational origin. J Kumamoto Med Soc 58 (1984) 56-70.

5. Takeya M, Imanishi $\mathbf{H}$, Takeuchi T. Electron microscopic study of polyneuropathy in VWF. Proc Jpn Pathol Assoc (in press).

6. Torii J, Kohsaka K, Imanishi H. Disturbance of peripheral nerves by use of chain saw: Observation of motor nerve conduction velocity. Jpn Med J 2829 (1978) 32-34.

7. Walton KW. The pathology of Raynaud's phenomenon of occupational origin. In: Taylor W, ed. The vibration syndrome. Academic Press Inc, London 1974 pp 109120. 